Physics International 3 (1): 1-8, 2012

ISSN 1948-9803

(C) 2012 Science Publications

\title{
Absolute Flux Comparison of Magnetospheric Particles
}

\author{
Miah M. Adel \\ Department of Chemistry and Physics, \\ Interdisciplinary Sciences Research Center, \\ University of Arkansas at Pine Bluff, Pine Bluff, AR 71601, USA
}

\begin{abstract}
Problem statement: The calculation of magnetospheric particle flux by dividing the particle count rate by the instrument geometric factor does not take into account the anisotropic pitch angle distribution function. A comparison of particle fluxes measured by different instruments fail to lead to the right comparison. To circumvent the omission of anisotropy of the pitch angle distribution and to make the correct comparison of particle fluxes, the instrument response function to different pitch angles within the sampling range of the instrument has been incorporated into the count rates over a certain readout time. Conclusion/Recommendations: A quantity in absolute comparison of magnetospheric particle flux has been found. The newly defined quantity of different observations does lead to the correct comparison for studying temporal variations. Investigators interested to study temporal features of magnetospheric particles over epochs may find the response functions for different instruments flown at different epochs useful for their study.
\end{abstract}

Key words: Magnetosphere, charged particles, particle telescope, count rate, flux, pitch angle, magnetic field, response function

\section{INTRODUCTION}

The charged particle detectors on board satellites may have conical shapes characterized by certain opening angle, a height and a sensitive base. The opening angle and the height are the major factors to detect particles of the desired pitch angle range. Along with the dependence on the pitch angle $(\alpha)$, the flux of magnetospheric particles is usually a function of Energy (E), magnetic field (B), McIlwain's parameter (L), latitude $(\lambda)$, (longitude (ǿ) and time $(\mathrm{t})$. The pitch angle distribution of magnetospheric particles which is usually in the form of $\sin ^{\mathrm{q}} \alpha$ is anisotropic in the sense that it does not indicate equal number of particles from equal intervals of pitch angles. But the calculation of the particle flux $j$ from the counting rate $\mathrm{N}$ instrument geometric factor and the energy interval $\Delta \mathrm{E}$ using the relation:

$$
\mathrm{j}=\mathrm{N} / \text { Geometric factor } \times \Delta \mathrm{E} \text { ) }
$$

Yields an isotropic flux in which $\mathrm{N}$ is independent of the direction of incidence and depends only on the size of the solid angle of acceptance. However, without taking into consideration the anisotropy factor, isotropic flux cannot be calculated. To study the temporal variation of magnetospheric fluxes, a comparison of measurements made at different epochs is required which urges the inclusion of the anisotropy factor with its observed range to calculate the flux.

The Geometric Factor (GF) in Eq. 1 does not include the pitch angle distribution function either (Sullivan, 1971). The pioneer investigators reported flux calculation using the above relation (Hovestadt et al., 1972; Moritz, 1972; Mizera and Blake, 1973; Scholer et al., 1975). All of this study relate to the observations of the equatorially mirroring particles. Equatorially mirroring particles $\left(\alpha_{\mathrm{e}}=90^{\circ}\right)$ peak the pitch angle distribution. However, the nonequatorially mirroring particles appear as fringes in the pitch angle spectrum.

This article describes how the pitch angle anisotropy can be included in the calculation of particle flux via the calculation of the instrument response function to particles of different pitch angles. Scientists working with old and currently operating satellite data will find the results very useful.

Response function: The observed counting rate of a detector telescope for a magnetospheric particle population in the pitch angle range $\alpha_{1}-\alpha_{2}$ and the energy range $E_{1}-E_{2}$ 
during a readout time interval $\mathrm{T}$ is given by the integral over the incoming particle direction $r$ of the product of the particle flux j (E, B. L $\alpha, \lambda$, ø, t) with the detector area A exposed normal to the incident direction i.e., (Miah et al., 1989; Miah, 1994) Eq. 2a:

$$
\mathrm{R}=1 / \mathrm{T} \int_{0}^{\mathrm{T}} \mathrm{dt} \int_{\mathrm{E}_{2}}^{\mathrm{E}_{2}} \mathrm{dE} \int_{\Omega} \mathrm{d} \varepsilon \int_{\mathrm{A}} \mathrm{dA} \cdot \mathrm{r}(\omega) . \mathrm{j}(\mathrm{E}, \mathrm{B}, \mathrm{L}, \alpha, \lambda, \phi, \mathrm{t})
$$

The particle is assumed to have the most general form of flux Eq. $2 b$ :

$$
\mathrm{j}(\mathrm{E}, \mathrm{B}, \mathrm{L}, \alpha, \lambda, \phi, \mathrm{t})=\mathrm{j}_{\mathrm{n}}(\mathrm{E}, \mathrm{B}, \mathrm{L}, \alpha, \lambda, \phi, \mathrm{t}) \mathrm{E}^{-\mathrm{b}} \sin ^{\mathrm{q}} \alpha
$$

where, $J_{n}$ is the normalization constant characteristic of the actual particle population, $E^{-b}$ is the energy spectrum and $\sin ^{q} \alpha$ is the pitch angle distribution function. The counting rate can then be written as Eq. 2c-2k:

$$
\mathrm{R}=\mathrm{j}_{\mathrm{n}}(\mathrm{E}, \mathrm{B}, \mathrm{L}, \alpha, \lambda, \phi, \mathrm{t}) 1 / \mathrm{T} \int_{0}^{\mathrm{T}} \mathrm{dt} \int_{\mathrm{E}_{1}}^{\mathrm{E}_{2}} \mathrm{E}^{-\mathrm{b}} \mathrm{dE} \int_{\alpha 1}^{\alpha 2} \mathrm{~d} \alpha \int_{\Omega} \mathrm{d} \omega
$$

$\int \mathrm{d} A \cdot r(\omega) \cdot \sin ^{\mathrm{q}} \alpha$

$$
=\mathrm{j}_{\mathrm{n}} \mathrm{QG}
$$

Where:

$$
\begin{aligned}
& Q=\int_{E 1}^{E 2} E^{-b} d E \\
& G=\int_{\alpha 1}^{\alpha 1} d \alpha \int_{\Omega} d \omega \int_{A} d A \cdot r(\omega) \cdot \sin ^{q} \alpha=A F
\end{aligned}
$$

With:

$$
F=\int_{\alpha 1}^{\alpha 2} F(\alpha) f(\alpha) d \alpha
$$

$\mathrm{F}(\alpha)=\sin ^{\mathrm{q}} \alpha$

And the efficiency functions:

$$
\begin{aligned}
& f(\alpha) \int_{\alpha 1}^{\alpha 2} \mathrm{~d} \alpha \int_{\Omega} \mathrm{d} \omega \int_{\mathrm{A}} \mathrm{d} A \cdot r(\omega) \\
& \mathrm{f}(\alpha) \int_{\alpha 1}^{\alpha 2} \mathrm{~d} \alpha \text { (geometric factor). }
\end{aligned}
$$

the geometric factor $=\int_{\Omega} \mathrm{d} \omega \int_{\mathrm{A}} \mathrm{dA} . \mathrm{r}$
Does not include the integration over the pitch angle distribution. The counting rate then reduces to Eq. 21:

$$
\mathrm{R}=\mathrm{AFJ}_{\mathrm{n}} \mathrm{Q}
$$

And the desired quantity for absolute comparison of magnetospheric particle flux is Eq. $2 \mathrm{~m}$ :

$$
\mathrm{J}_{\mathrm{n}}=\mathrm{R} / \mathrm{AFQ}
$$

The above equations (2a) through $(2 \mathrm{~m})$ are just defining equations for what they stand and do not need any discussion. The limits of the integrations in the equations pertaining to an instrument will be instrument specific.

The acceptance cone of the telescope allows particles of a wide pitch angle spectrum to enter the detector. The relative efficiency of a detector for a given pitch angle $\alpha$ is defined as the fraction of the associated space angle intercepted by the telescope cone, duly weighted by the fraction of the total area which is perpendicular to the incoming particle beam and normalized by the efficiency of a halfomnidirectional detector.

The details of the steps leading to the efficiency calculation are presented in another article (Adel, 2008). Briefly, the detector area is divided into a number of equal elemental Areas $(\Delta \mathrm{A})$. The center point of each $\Delta \mathrm{A}$ is taken as the representative point of that segment. A telescope cone which is right circular only for the central point of the circular sensitive base and elsewhere just a circular, is imagined with the apex at this point. The pitch angle range is determined for this telescope cone. The response function for all pitch angles within the range is calculated for this representative point. The steps are repeated for all other representative points and then averaged over all segments. Next, coordinate transformations are used to define, in the telescope frame, the direction of the geomagnetic field. Then, a semi-analytic computer algorithm determines the points of intersection of the telescope and the pitch angle cones. Finally, the results are integrated over the pitch angle distribution and averaged over the entire detector area.

Setup of the pitch angle and telescope frames of observation: The above formulation related to the geometry of a conical particle telescope may be understood with the help of Fig. 1. Both the pitch angle cone and the telescope cone are shown in the figure. The pitch angle frame is set up in such a way as $\mathrm{Z}^{\prime}$ axis points along the $\mathrm{B}$ direction which is also the axis of the pitch angle cone. $\mathrm{X}^{\prime}$ is perpendicular to $\mathrm{B}$ and lies in 
the plane of $\mathrm{B}$ and the telescope axis; the $\mathrm{Y}^{\prime}$ axis is chosen to make the system right-handed. The telescope cone is shown with the apex at an off-centered position of the sensitive base area of the detector. At this location, it is just a circular cone. Its coordinate system is XYZ. OF is the telescope cone axis. The axes of the two cones are along their respective Z-axes. $\chi^{\prime \prime}$ is the angle between the two cone axes. The telescope intercepts the arc $\mathrm{CD}$ of the pitch angle cone. The telescope axis always lie in the $\mathrm{Z}^{\prime} \mathrm{X}^{\prime}$ plane of the pitch angle frame so that the azimuth angle measured from this plane is zero. Further, in the pitch angle frame, the direction of any incident particle is specified by the polar angle $\alpha$ and an azimuth angle $\beta^{\prime \prime}$ measured from the $Z^{\prime} Z^{\prime} X^{\prime}$ plane, positive on one side of it $\left(\beta_{1}{ }^{\prime \prime}\right)$ in the counterclockwise direction and negative on the other side $\left(\beta_{2}{ }^{\prime \prime}\right)$ in the clockwise direction.

Calculation of azimuth Angle $\beta$ " in the pitch angle frame: From unit vectors directed along OA, OC, OD and $\mathrm{OF}$ in the pitch angle frame, we can find the angles $\beta_{1}{ }^{\prime \prime}$ and $\beta_{2}{ }^{\prime \prime}$. $\beta_{1}{ }^{\prime \prime}$ is the angle between the plane formed by OA and OC and the plane formed by $\mathrm{OA}$ and OF. And $\beta_{2}{ }^{\prime \prime}$ is the angle between the plane formed by $\mathrm{OA}$ and $\mathrm{AD}$ and the plane formed by $\mathrm{OA}$ and $\mathrm{OF}$. Cross products of unit vectors directed along $\mathrm{OA}$ and $\mathrm{OC}$ will be perpendicular to their plane and the cross product of the unit vectors directed along $\mathrm{OA}$ and $\mathrm{AF}$ will be perpendicular to their plane. The dot product of these unit vectors perpendicular to their respective planes yields $\beta_{1}{ }^{\prime \prime}$. We can similarly form the cross product of unit vectors directed along OA and $\mathrm{OD}$ and the cross product of the unit vectors directed along OA and OF. The dot product of these two cross products yields the angle $\beta_{2}{ }^{\prime \prime}$. The coordinates of the points $\mathrm{C}$ and $\mathrm{D}$ of intersections of the telescope and pitch angle cones are found by an iterative method described below.

The iterative process: A given pitch angle within the instrumental pitch angle range $\chi^{\prime \prime}-\alpha$ and $\chi^{\prime \prime}+$ angle EOF is selected. The two points of intersections of the pitch angle cone with telescope cone are found by iteration setting the difference between the selected pitch angle and the computed pitch angle $\Delta \alpha<0.001^{\circ}$. The intersection of the opening of the pitch angle cone and the plane of the opening of the telescope cone is a conic section having the projection of the magnetic field vector on the plane of opening ring of the telescope as the axis of symmetry. In the iterative process, the two points of intersection are sought on either side of the projection of the magnetic field vector on the opening ring of the telescope.
Figure 2 is helpful in determining the $\mathrm{X}, \mathrm{Y}, \mathrm{Z}$ coordinates of the points of intersections. The chord PO'Q represents the projection of the magnetic field vector onto the plane of the opening ring of the telescope. OP is the radius of the ring and $\mathrm{OO}^{\prime}$ is equal to $\mathrm{a}$, the shift of the origin along the $\mathrm{X}$-axis.

The angle $\chi$ at the observation point is determined from the component of the magnetic field Eq. 3a:

$$
\chi=\cos ^{-1}\left(\mathrm{~B}_{\mathrm{x}} /\left(\mathrm{B}_{\mathrm{x}^{2}}+\mathrm{B}_{\mathrm{y}^{2}}\right)^{1 / 2}\right)
$$

supplied by the magnetic field model.

Each time a $\Delta \chi$ is added $\chi, \mathrm{X}, \mathrm{Y}$ and $\mathrm{Z}$ coordinates are determined Eq. $3 b$ :

$\mathrm{X}=\mathrm{R} \cos \chi^{\prime}-\mathrm{a} ; \mathrm{Y}=\mathrm{R} \sin \chi ; \mathrm{Z}=\mathrm{H}-1.7336 \mathrm{~cm}$

where $\chi^{\prime}$ can be obtained from Eq. 3c:

$\mathrm{PO}^{-2}=\mathrm{R}^{2}+\mathrm{a}^{2}-2 \mathrm{aR} \cos \chi$

And:

$\mathrm{PO}^{-2}=\mathrm{R}^{2}+\mathrm{a}^{2}-2 \mathrm{POO}^{\prime} \cos (180-\chi)$

The detector dimensions used in this study pertains ONR-602 experiment (Miah, 1991a). As said above, Eq. 3a relates to the magnetic field model of the Earth, and the Eq. 3b through 3d relate to the geometry involved in the calculation.

Unit vector along the incident direction is calculated from X., Y and Z coordinates. A dot product of this vector with the unit magnetic field vector gives the pitch angle. Then $\Delta \alpha$ is calculated. The above steps are repeated until $\Delta \alpha$ falls lower than 0.001 degree. The entire operation is repeated on the other side of the magnetic vector position and the second point of intersection is found for the pitch angle under consideration.

Accuracy of finding the points of intersections: A check was made to see if the two points of intersections satisfy both the pitch angle and the telescope cone equations. For this purpose, the following equation for the telescope cone was deduced with the cone apex at $(\mathrm{a}, 0,0) \mathrm{Eq} .4 \mathrm{a}$ :

$$
\left(\mathrm{X}+\mathrm{H} \tan \gamma_{1}\right)^{2} \mathrm{Y}^{2}=\mathrm{H}^{2}\left(\tan \left(\gamma_{1}+\gamma_{2}\right)-\tan \gamma_{1}\right)^{2}
$$

In Fig. 3, angle $\mathrm{O}^{\prime} \mathrm{O}^{\prime \prime} \mathrm{O}=\gamma_{2}$ and the angle $\mathrm{OO}^{\prime \prime} \mathrm{P}^{\prime \prime \prime}=\gamma_{1}$. The equation can be simplified to Eq. $4 \mathrm{~b}$ :

$$
\mathrm{X}^{2}+\mathrm{Y}^{2}+2 \mathrm{aX}-2(\mathrm{R}+\mathrm{a})(\mathrm{R}-\mathrm{a})=0
$$

where, in the figure, $a=O^{\prime}\left(=\mathrm{O}^{\prime \prime} \mathrm{O}^{\prime \prime}\right)$, the distance from the central point of the detector to the telescope cone and $\mathrm{OP}=\mathrm{R}$, the radius of the mouth of the telescope cone. 


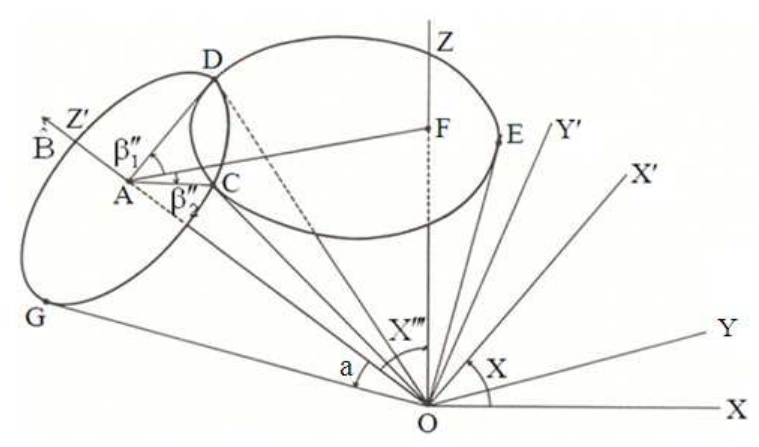

Fig. 1: Illustrations of the points of intersections of telescope cone CDEO and the pitch angle cone $\mathrm{CDGO}$ at points $\mathrm{C}$ and $\mathrm{D}$. Their axes are inclined at an angle $\chi^{\prime \prime \prime} . X^{\prime} Y^{\prime} Z^{\prime}$ are the pitch angle cone axes and $\mathrm{XYZ}$ are the telescope cone axes. $\mathrm{X}^{\prime} \mathrm{ZZ}^{\prime}$ lie in one plane. $\chi$ is the angle between the $X$-axis and the projection of the magnetic field or the projection $\mathrm{X}^{\prime} \mathrm{ZZ}$ ' plane in the XY-plane

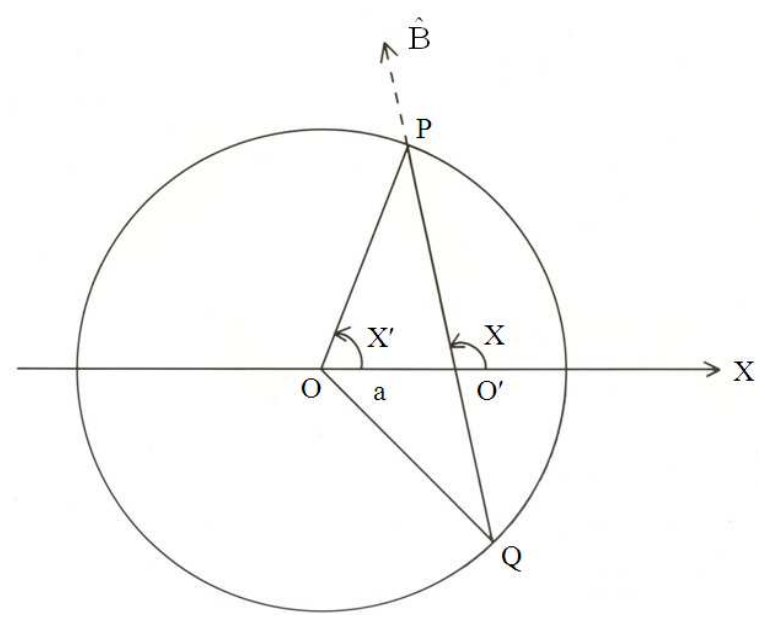

Fig. 2: Illustration of finding the $\mathrm{X}, \mathrm{Y}$, and $\mathrm{Z}$ coordinates of the points of intersection of the pitch angle and telescope cones

The pitch angle cone equation is written with the magnetic field vector as the axis. Later, through transformation of coordinates, the equation is obtained in the telescope cone coordinate system. The transformation equations used are given below. The equation of the pitch angle cone with the axis along the magnetic field is Eq. $4 \mathrm{c}$ :

$$
\mathrm{Y}^{2}+\mathrm{Z}^{2}=\mathrm{X}^{2} \tan ^{2} \alpha
$$

where, $\alpha$ is the given pitch angle. The first rotation done is a clockwise vector rotation around the OZaxis through $\chi$ given in terms of the magnetic field components Eq. $4 \mathrm{~d}$ :

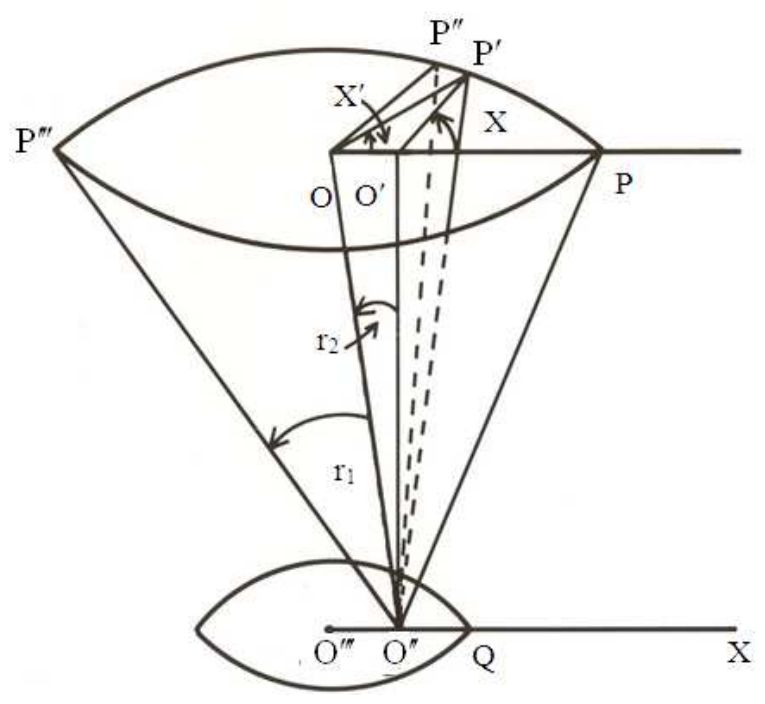

Fig. 3: Illustrate the telescope cone equation when the apex is at an off-centered point on the sensitive base. At this point, it is just a circular cone

$\chi=\cos ^{-1}\left(\mathrm{~B}_{\mathrm{x}} /\left(\mathrm{B}_{\mathrm{x}}{ }^{2}+\mathrm{B}_{\mathrm{y}}\right)^{2}\right)^{0.5}$

The components of the rotated vectors are Eq. 4e:

$\mathrm{X}^{\prime}=\mathrm{X}^{\prime} \operatorname{cox} \chi+\mathrm{Y}^{\prime} \sin \chi$
$\mathrm{Y}^{\prime}{ }^{\prime}=-\mathrm{X}^{\prime} \sin \chi+\mathrm{Y}^{\prime} \cos \chi$
$\mathrm{Z}^{\prime}{ }^{\prime}=\mathrm{Z}$

The next rotation is around $O Y^{\prime}$ axis through $\chi^{\prime \prime}$ $=90-\chi^{\prime \prime \prime}$, the angle between the magnetic field vector and the $\mathrm{Z}$-axis, in the anticlockwise direction.

The rotation yields Eq. 4f:

$$
\begin{aligned}
& X^{\prime \prime}=X^{\prime \prime} \cos \chi{ }^{\prime}+Z^{\prime} \sin \chi{ }^{\prime} \\
& Y^{\prime \prime}=Y^{\prime} \\
& Z^{\prime \prime}=-X^{\prime}{ }^{\prime} \sin \chi^{\prime \prime}+Z^{\prime}{ }^{\prime} \cos \chi{ }^{\prime}
\end{aligned}
$$

Equation $4 \mathrm{c}, 4 \mathrm{e}$ and $4 \mathrm{f}$ define the pitch angle cone in the telescope cone frame.

Substitution of the coordinates of the two points of intersections found by the iteration satisfies the telescope cone equation exactly. However, the pitch angle cone equation yields a value of $\sim 1 \times 10^{-4}$ for pitch angles which are not equal to $90^{\circ}$. For $90^{\circ}$ angles $\chi^{\prime \prime}$ yields $\sim 1 \times 10^{-6}$. The accuracy of the solution depends upon the condition that the difference between the given and the computed pitch angles should be less than $0.001^{\circ}$. 
Phy. Intl. 3 (1): 1-8, 2012

Efficiency calculation in the pitch angle frame: The integral for $\mathrm{G}$ can be written as Eq. 5a:

$$
G=\int_{\alpha 1(\mathrm{dA})}^{\alpha 2(\mathrm{dA})} \mathrm{d} \alpha \int_{\mathrm{d} \Omega} \mathrm{d} \omega \int_{\mathrm{A}} \mathrm{dA} \cdot \mathrm{r}(\omega) \cdot \sin ^{\mathrm{q}} \alpha
$$

where, $\mathrm{d} \Omega$ is the domain of the solid angle $\omega$ and is determined from the pitch angle cone and the telescope cone geometry, $\mathrm{d} \omega=\sin \alpha \mathrm{d} \beta$ ',,$\alpha$ being the polar angle and $\beta$ ', the azimuth angle. The perpendicular component of the area element is dA.r $(\omega)$ looking into $\omega$. It may be understood that $G$ is a product of the detector sensitive area perpendicular to the incoming particle beam, an effective solid angle and the pitch angle distribution. Its unit is $\mathrm{cm}^{2}$.sr Eq. 5b:

$$
\alpha_{2}(\mathrm{dA}) \quad \beta{ }^{\prime}{ }_{\max }\left(\alpha, \mathrm{dA}, \chi \chi^{\prime \prime}\right)
$$

$$
\mathrm{G}=\int \sin ^{\mathrm{q}} \alpha \mathrm{d} \alpha \int \sin \alpha \mathrm{d} \beta, \int \mathrm{dA} \cdot \mathrm{r}(\omega)
$$

$\alpha(\mathrm{dA}) \beta,{ }^{\prime \prime}\left(\alpha, \mathrm{dA}, \chi^{\prime, \prime}\right) \mathrm{A}$

The integral cannot be evaluated analytically. However, it can be evaluated semi-analytically as follows.

The incident vector in the pitch angle frame is given by Eq. $5 c$ :

$\mathrm{r}=\sin \alpha \cos \beta “+\sin \alpha \sin \beta^{\prime} \mathrm{j}+\cos \alpha \mathrm{k}$

The vector components along $\mathrm{dA}$ in the pitch angle frame are given by Eq. 5d:

$\mathrm{dA}=\sin \chi{ }^{\prime \prime} \mathrm{i}+0 \mathrm{j}+\cos \chi{ }^{\prime \prime} \mathrm{k}$

The last integral is Eq. 5e and 5f:

$$
\begin{aligned}
& \int_{\mathrm{A}} \mathrm{dA} \cdot \mathrm{r}(\omega) .=\int_{\mathrm{A}} \mathrm{dA}\left(\sin \alpha \cos \beta “ \sin \chi^{\prime \prime}+\cos \alpha \cos \chi^{\prime \prime}\right) \\
& \quad \mathrm{n} \\
& \approx \sum_{\mathrm{i}=1} \Delta \mathrm{A}_{\mathrm{i}}\left(\sin \alpha_{\mathrm{i}} \cos \beta{ }^{\prime}{ }_{\mathrm{i}} \sin \Delta \chi \chi^{\prime,}+\cos \alpha_{\mathrm{i}} \cos \chi{ }^{, \prime \prime}\right)
\end{aligned}
$$

The entire angular integration is then Eq. $5 \mathrm{~g}$ and $5 \mathrm{~h}$ :

$$
\begin{aligned}
& \alpha_{2}(\mathrm{dA}) \quad \beta{ }^{\prime}{ }_{\text {max }}\left(\alpha, \mathrm{dA}_{\mathrm{i}}, \chi{ }^{\prime,},\right) \\
& \mathrm{G}=\int \sin ^{\mathrm{q}} \alpha \mathrm{d} \alpha \int \sin \alpha \mathrm{d} \beta, \quad \int \mathrm{dA} \cdot \mathrm{r}(\omega) \\
& \alpha_{1}(\mathrm{dA}) \quad \beta,{ }_{\text {min }}\left(\alpha, \mathrm{dA}_{\mathrm{i}}, \chi,{ }^{\prime \prime}\right) \mathrm{A} \\
& \beta_{\text {max }}^{\prime \prime}\left(\alpha_{\mathrm{ij}}, \mathrm{dA}_{\mathrm{i}}, \chi^{\prime \prime}\right) \\
& =\sum_{\mathrm{j}=1}^{\mathrm{m}} \sin ^{\mathrm{q}}\left(\alpha_{\mathrm{j}}\right) \Delta \alpha \sum_{\mathrm{i}=1}^{\mathrm{n}} \Delta \mathrm{Ai} \int \mathrm{d} \beta{ }^{\prime \prime}\left(\sin ^{2} \alpha_{\mathrm{ij}} \cos \beta^{\prime \prime} \sin \chi^{\prime \prime}\right) \\
& \beta_{\text {min }}^{\prime \prime}\left(\alpha_{i j}, \mathrm{dA}_{\mathrm{i}}, \chi^{\prime \prime \prime}\right)
\end{aligned}
$$

where, $\Delta \alpha=\left(\alpha_{2}-\alpha_{1}\right) / \mathrm{m}$ is in radian units Eq. $5 \mathrm{i}-5 \mathrm{k}$ :

$$
\begin{aligned}
& =\mathrm{A} \sum_{\mathrm{j}=1}^{\mathrm{m}} \sin ^{\mathrm{q}}\left(\alpha_{\mathrm{j}}\right) \Delta \alpha \cdot \sum_{\mathrm{i}=1}^{\mathrm{n}}\left(\Delta \mathrm{A}_{\mathrm{i}} / \mathrm{A}\right)\left[\operatorname { s i n } ^ { 2 } \alpha _ { \mathrm { ij } } \chi ^ { \prime \prime \prime } \left(\sin \beta_{\text {max }}^{\prime \prime}\right.\right. \\
& \left.\left.\sin \beta_{\text {min }}\right)+\sin \alpha_{\mathrm{ij}} \cos \alpha_{\mathrm{ij}} \operatorname{cox} "^{\prime \prime}\right) \\
& \mathrm{x}\left(\sin \beta^{\prime \prime}{ }_{\max }-\sin \beta_{\text {min }}\right) \\
& =\mathrm{A} \cdot \sum_{\mathrm{j}=1}^{\mathrm{m}} \mathrm{F}\left(\alpha_{\mathrm{j}}\right) \Delta \alpha \mathrm{f}\left(\alpha_{\mathrm{j}}\right)
\end{aligned}
$$

Where:

$$
\begin{aligned}
& \mathrm{f}\left(\alpha_{\mathrm{j}}\right)=(1 / 2 \pi) \sum_{\mathrm{i}=1}^{\mathrm{n}}\left(\Delta \mathrm{A}_{\mathrm{i}} / \mathrm{A}\right)\left[\operatorname { s i n } ^ { 2 } \alpha _ { \mathrm { ij } } \operatorname { s i n } \mathrm { x } { } ^ { \prime \prime } \left(\sin \beta^{\prime \prime}{ }_{\max }-\right.\right. \\
& \left.\left.\sin \beta_{\text {min }}^{\prime \prime}\right)+\sin \alpha_{\mathrm{ij}} \cos \alpha_{\mathrm{ij}} \cos \chi^{\prime \prime \prime}\right) \\
& \left.\mathrm{x}\left(\sin \beta^{\prime \prime}{ }_{\text {max }}-\sin \beta^{\prime \prime}{ }_{\text {min }}\right)\right]
\end{aligned}
$$

is the efficiency function for $\alpha_{j}$ applied to the whole detector Eq. 51 and $5 \mathrm{~m}$ :

$\mathrm{G}=\mathrm{AF}$

where:

$$
F \sum_{j=1}^{n} F\left(\alpha_{j}\right) \Delta \alpha f\left(\alpha_{j}\right)
$$

F can be evaluated by the simple trapezoid rule. It has the units of steradian. The detector count rate is then given by Eq. 5n:

$$
\mathrm{R}=\mathrm{AFJ}_{\mathrm{n}} \mathrm{Q}
$$

Comparison of numerical and analytical results: Several tests were carried out for the efficiencies of both the point and the whole detector for $90^{\circ}$ pitch angle with magnetic field configuration in the plane of the detector because the analytical tests can be done only for $90^{\circ}$ pitch angles. The checked situations correspond to $\lambda=0^{\circ}, \varphi=90^{\circ}$ and $\delta=0^{\circ}$.

Fractional efficiency for $90^{\circ}$ pitch angle particles is easily checked for the central point of the detector to be equal to the space angle intercepted by the diameter of the opening ring at the detector point, multiplied by the fractional area represented by that point and exposed normal to the particles of pitch angle $90^{\circ}$. The calculated and computed values of response functions show no difference for this particular point. The response function calculated for the same pitch angle was done at two other points $(0.28194,0.0 \mathrm{~cm})$ and $(0.46990,0.0 \mathrm{~cm})$ on the detector, where the radius vector was divided into 3 equal parts producing 9 equal elementary representative areas. No differences were found between the computed and analytically calculated results. 
Magnetic field dependence of the response function: As to the influence by the activity of the Earth's magnetosphere, the response function calculated depends upon the magnetic field orientation. Magnetospheric activities are influenced by the solar conditions. The response function thus depends on the solar conditions. Whereas no calculations were made for solar quiet and violent conditions, some differences were observed between the response functions calculated for the dipole field and the real geomagnetic field. In the dipole field, the peak efficiency was for $\alpha_{e}$ $=90^{\circ}$. But in the real field geomagnetic equator, the response function peaks for $\alpha_{e}=90 \pm 7^{\circ}$. This demonstrates the influence of the magnetic field variation, a magnetospheric activity.

Normalization constant $\mathbf{J}_{\mathbf{n}}$ : The normalization constant can be found from the above equation as Eq. 6:

$$
\mathrm{J}_{\mathrm{n}}=\mathrm{R} / \mathrm{AFQ}
$$

At the equator $\lambda=0$ and $\mathrm{B} \infty 1 \mathrm{~L}^{-3}$. For low altitude satellite passes $(300-456 \mathrm{~km})$, the range of $\mathrm{L}$ is very small. This means the dependence of $J_{n}$ upon $\mathrm{B}$ an $\mathrm{L}$ is very small. The dependence of $\mathrm{J}_{\mathrm{n}}$ upon the longitude $\varphi$ is insignificant at the equator. So, the variation of $J_{n}$ is principally with the exponent $q$ of the pitch angle function and the time or epoch $t$. Taking a range of values for $\mathrm{q}$ (Miah, 1991b; Miah et al., 1992), we can study the variation of $\mathrm{J}_{\mathrm{n}}$. with time or epoch. $J_{n}$ is representative of a particle population and the comparison of $\mathrm{J}_{\mathrm{n}}$ for different epochs is basically a comparison of particle population for different epochs. Multidimensional plots can be produced to study the variation of $\mathrm{J}_{\mathrm{n}}$ upon other factors in their applicable ranges.

\section{Application to measured data:}

Pitch angle distribution function. As to the area of applicability, the method is applicable at any latitude and longitude. To show it qualitatively, we can pick up only the equatorial region for the equatorially mirroring particles for which we know the pitch angle $\left(\alpha_{e}\right)$ distribution function. It has the form $\sin ^{\mathrm{n}} \alpha_{\mathrm{e}}$ where $n$ runs from 5-21 from different observations (Moritz, 1972; Mizera and Blake, 1973; Daiem, 2010a; 2010b; Sing et al., 2010; Yusif et al., 2010) with the most likely value in the middle of the range (Miah et al., 1992). Sampling function at the equator can be approximated by the same sine function with exponent 13. So the detector can respond to all the equatorially mirroring particles. For off-equatorially mirroring particles at the equator, it cannot detect any particles beyond the equatorial pitch angle $90^{\circ} \pm 37^{\circ}$.
If we know the pitch angle distribution function of particles mirroring at other latitudes, we can check with the applicability of the method with the instrument response function calculated at that latitude.

Application to old satellite data: Regarding an application example to a specific instrument and observation data, the method developed was used to calculate the response functions of the monitor telescope in the ONR-602 Experiment and the German Research Satellite Azur. Figure 4 shows the sampling efficiency as a function of the pitch angles at the dipole equator. Azur telescope axis was within $90 \pm 5^{\circ}$ with the magnetic field at the equator. Its low opening angle accounts for its low values of response function.

In Eq. 6, $\mathrm{J}_{\mathrm{n}}$ depends on the exponent $\mathrm{q}$ of the pitch angle distribution function and the epoch or time $\mathrm{t}$ (Miah et al., 1992). Considering a range of values for $\mathrm{q}$, we can study the variation of $J_{n}$ with time t. Since $J_{n}$ is representative of the particle population, comparison of $\mathrm{J}_{\mathrm{n}} \mathrm{s}$ for different epochs is basically a comparison of the particle population at different epochs. In Fig. 5, the open circles represent the $J_{n}(h, t)$ values for the monitor detector flux in ONR-602 Experiment on board S81-1 mission observed at $277 \mathrm{~km}$ and the squares represent the same values for Azur for measurements at $450 \mathrm{~km}$. For clarity, squares and circles have been offset along the horizontal scale. Within the uncertainties, the fluxes measured by Moritz (1972) at $450 \mathrm{~km}$ and by the monitor detector at $277 \mathrm{~km}$ are indistinguishable. Altitude difference and altitude-dependent source depletion model can explain why the fluxes from the two measurements are indistinguishable (Miah, 1988).

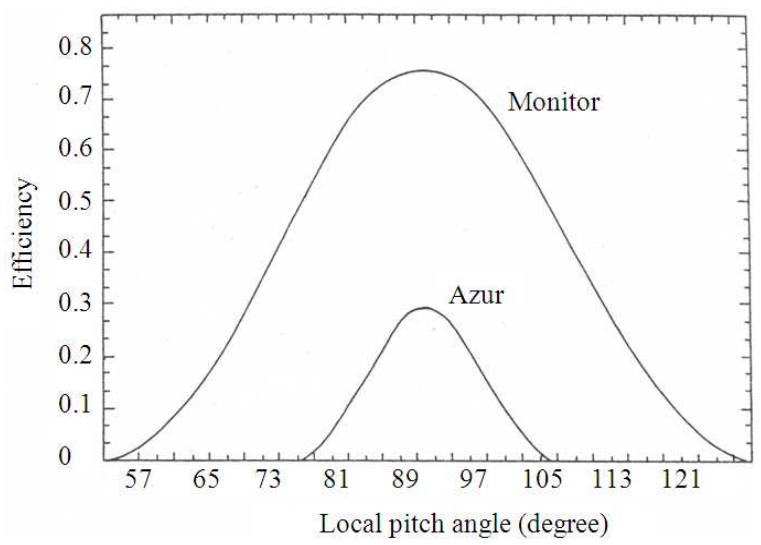

Fig. 4:Illustrates the response functions of the Azur telescope on board the German Research Satellite and the monitor telescope on board US Air Force S81-1 mission 


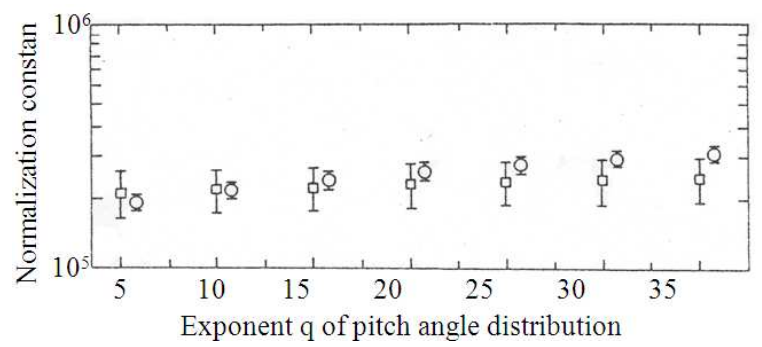

Fig. 5: Illustrates the comparison of absolute fluxes measured by the Azur telescope on board the German Research Satellite and the monitor telescope on board US Air Force S81-1 mission for different values of $\mathrm{q}$, the pitch angle distribution function experiment. The circles represent the $J_{n}(h, t)$ for the monitor telescope and the squares for the Azur telescope

Applications to new mission data: The method can be used with current as well as future instruments. The only requirement is an acceptance cone and a sensitive base. As to the application with new instruments on board satellites, Oersted may be selected. It has a charged particle detector (Cabera et al., 2005). The details of the calculation of the response function taking consideration of the satellite orbital characteristics and the detector orientation and geometry will be reported in a future study. Regarding the comparison of absolute flux, an uncertainty in the altitude dependence of particle flux will remain unsolved since this dependence is not known for the altitude range of 450 $\mathrm{km}$, the Azur's observation altitude, to $640 \mathrm{~km}$, Oersted's observation altitude. However, the same uncertainty has remained in Fig. 5 in the comparison of absolute fluxes between Azur's and monitor's observations because of the lack of observed flux data in the altitude range interval of $277-450 \mathrm{~km}$. It is of future interest to explore the application of this geometrical method to calculate the instrumental response function to non-charged particle radiation.

\section{CONCLUSION}

The instrument response function to particles of different pitch angles naturally plays the part in the instrumental counting rate. The simple flux calculation from the counting rate, geometric factor and the energy interval does not incorporate this factor. The writing of the counting rate over a certain time interval shows explicitly the pitch angle-related response function. The concept of the product function of the effective detector sensitive area perpendicular to the incoming particle beam, the effective solid angle and the pitch angle distributions is very useful in the definition of the relative detector efficiency, for a given pitch angle $\alpha$, as the fraction of the associated space angle intercepted by the telescope cone, duly weighted by the fraction of the total area which is perpendicular to the incoming particle beam and normalized by the efficiency of a half-omni-directional detector. The semi-analytical method developed in this study can be applied to any particle telescope having some acceptance angle and some sensitive base. The efficiency function can be calculated to any degree of accuracy. The normalization constant becomes independent of detector geometry and its comparison for different epochs is basically the comparison of absolute flux for those times. It is planned to explore the use of the geometric treatment presented here to non-charged particle radiation.

\section{REFERENCES}

Adel, M.M., 2008. A detector telescope's pitch angle sampling of magnetospheric particles. Earth, Planets Space, 60: 753-761.

Cabera, J., M. Cyamukungu, P. Stauning, A. Leonov and P. Leleux et al., 2005. Fluxes of energetic protons and electrons measured on board the Oersted satellite. Ann. Geophys., 23: 2975-2982.

Daiem, A.A., 2010a. Characteristics of compound multiplicity in ${ }^{24} \mathrm{Mg}$ emulsion at $4.5 \mathrm{GeV} / \mathrm{c}$, Phys. Int., 31-37. DOI: 10.38844/pisp.2010.31.37

Daiem, A.A., 2010b. Featured study on target fragmentation in the collision of $24 \mathrm{Mg}$ and $28 \mathrm{Sr}$ with emuslion nuclei $3.7 \mathrm{GeV}$, Phys. Int., 45-52. DOI: $10.3844 /$ pisp.2010.45.52

Hovestadt, D., B. Hausler and M. Scholer, 1972. Observation of energetic particles at very low altitudes near the geomagnetic equator. Phys. Rev. Lett., 28: 1340-1344.

Miah, M. A., 1994. Significant variation of proton population in the equatorial thermosphere. Adv. Space Res., 14: 229-232.

Miah, M.A., 1988. Global Zones of Particle Precipitation. Ph. D. Thesis, Department of Physics and Astronomy, Louisiana State University, Baton Rouge.

Miah, M.A., 1991a. The ONR-602 Experiment and investigation of particle precipitation near the equator. J. Geomagnetism Geoelec., 43: 445-460.

Miah, M.A., 1991b. Observation of $\mathrm{Z} \geq 1$ particles below $300 \mathrm{~km}$ near the geomagnetic equator. J. Geomagnetism Geoelec., 43: 461-475.

Miah, M.A., J.W. Mitchell and J.P. Wefel, 1989. Magnetospheric particle detection efficiency of a conical telescope. Nucl. Instr. Meth. Phys. Res., 281: 622-627. DOI: 10.1016/0168-9002(89)91499$\mathrm{X}$ 
Miah, M.A., K. Nagata, T. Kohno, H. Murakami and A. Nakamoto et al., 1992. Spatial and temporal features of $0.64-35 \mathrm{MeV}$ protons in the space station environment: EXOS-C observations, J. Geomagnetism Geoelec., 44: 591-610.

Mizera, P.F. and J.B. Blake, 1973. Observations of ring current protons at low altitudes. J. Geophys. Res., 78: 1058-1062. DOI: 10.1029/JA078i007p01058

Moritz, J., 1972. Energetic protons at low equatorial altitudes. Zeitschrift fur Geophysik, Band, 38: 701-717.

Scholer, M., D. Hovestadt and G. Morfil, 1975. Energetic $\mathrm{He}+$ ions from the radiation belt at low altitudes near the geomagnetic equator. J. Geophys. Res., 80: 80-85. DOI: 10.1029/JA080i001p00080
Sing, M.K., A.K. Soma, R. Pathak and V. Sing, 2010 Two source emission behavior of helium projectile in ${ }^{84} \mathrm{Kr}$ interaction at around $1 \mathrm{GeV}$ per nucleon. Phys. Int. 1 : 95-101. DOI: 10.3844/pisp.2010.109.115

Sullivan, J.D., 1971. Geometrical factor and directional response of single and multi-element particle telescopes. Nucl. Instruments Meth., 95: 5-11. DOI: 10.1016/0029-554X(71)90033-4

Yusif, A.K., I.B. Bahari and M.S. Yasin, 2010. Identifications of the specific relations among physical parameters which use to quantify interaction effect of charged particles at low dose. Phys. Int., 1: 90-94. DOI: 10.3844/pisp.2010.94.98 